\title{
Floral behaviour, flowering phenology and fruit production of passion fruit (Passiflora Species) in East Malaysia
}

\begin{abstract}
Passiflora species generally known as passion fruit may well be the most fascinating plant of the tropics. This plant is known to be self-incompatible in its native environment and it affects the fruit production. Extend of passion fruit set is highly dependent on effective pollinators such as carpenter bees and honey bees. However, in East Malaysia, there was good fruit sets, although lesser number of pollinators visiting the farm or sometimes absence. It is important therefore, to study the reproduction mechanisms of Passiflora plants, because this factor reflect the quantity of the fruit produced. The present studies aim therefore to evaluate the floral behaviours, reproductive mechanism and fruit production of locally grown Passiflora species. Based on the observations, Passiflora species was classified as "steadystate species" that exhibit constant production of few flowers, each day lasting only one day. All species have the ability to adapt to the local condition where plants continuously grew and produced flowers and fruits all the year around. The flowering and fruiting were not synchronized and showed a marked phenological pattern with species. Good fruit yields were observed throughout the year which was attributed to Passiflora species ability for self-pollination, involving the movement of styles to lead the stigma faced the anthers during flower blooming. This is an important phenomenon in order to reduce the distance between the stigma and anther, aid for pollination and provided reproductive assurance to the species. As P. edulis growing in its native environment is reported to be self-incompatible and have become selfcompatible in the present study; showed that evolution of these features is an important factor for its adaptability and survivability, thus crucial to P. edulis propagation optimally in local conditions.
\end{abstract}

Keyword: Self-pollination; Fruit sets; Herkogamy; Homogamous; Passion fruit 